\title{
Physiological response of glutamine and glutamic acid supplemented broiler chickens to heat stress
}

\begin{abstract}
This study was conducted to investigate the effects of dietary glutamine (Gln) + glutamic acid (Glu) supplementation on growth performance and physiological stress response broilers exposed to cyclic heat stress. Day-old commercial broiler chickens were kept in environmentally controlled chambers and fed (i) basal diet (control), (ii) basal diet $+0.5 \%$ Gln+Glu from 1 to 21 days of age. From day 22-42, chicks in one chamber were subjected to $5 \mathrm{~h}$ daily heat stress at $34 \pm 1^{\circ} \mathrm{C}$ and the other chicks remain in thermoneutral condition. Weight gain and FCR significantly improved during the starter (day 1-21) and overall (day 1-42) periods with Gln+Glu supplementation when compared to control. Supplementing birds with Gln+Glu has also significantly reduced mortality rate in birds under heat stress. Physiologically, provision of Gln+Glu for the birds under heat stress resulted in higher heat shock protein 70 (Hsp70) and ovotransferrin (OVT) production. In conclusion, it appears that the beneficial effect of Gln+Glu supplementation in performance and survivability of broiler under heat stress may be partially due to enhancement of Hsp70 and OVT synthesis.
\end{abstract}

Keyword: Broiler, nutrition; Heat stress; Glutamine; Glutamic acid; Acute phase protein; Hsp70 